\title{
The Economic Impact of Levothyroxine Dose Adjustments: the CONTROL HE Study
}

\author{
Frank R. Ernst ${ }^{1} \cdot$ Peri Barr $^{1} \cdot$ Riad Elmor $^{1} \cdot$ Walter Sandulli $^{2} \cdot$ Lionel Thevathasan $^{3,4}$. \\ Arnold B. Sterman ${ }^{5} \cdot$ Jessica Goldenberg $^{2} \cdot$ Kevin Vora ${ }^{2}$
}

Published online: 31 October 2016

(c) The Author(s) 2016. This article is published with open access at Springerlink.com

\begin{abstract}
Background In general, hypothyroidism can be adequately treated with a consistent daily dose of levothyroxine. However, the need for levothyroxine dose adjustments is frequent in clinical practice. The extent to which levothyroxine dose adjustments increase the utilization of healthcare resources has not previously been described in the clinical literature.

Objective The primary objective of our study was to measure the effect of levothyroxine dose adjustments in terms of their utilization of healthcare resources including direct and indirect costs. A secondary goal was to identify any differences in patient characteristics that may be responsible for levothyroxine dose adjustments.

Methods A retrospective medical chart review was conducted among patients of selected healthcare providers in the USA. Patients who were recently started on levothyroxine therapy ( $<6$ months) were excluded to avoid
\end{abstract}

Electronic supplementary material The online version of this article (doi:10.1007/s40261-016-0462-3) contains supplementary material, which is available to authorized users.

Frank R. Ernst

frankrernstrx@gmail.com

1 Health Economics and Outcomes Research, Indegene, Inc., 222 Chastain Meadows Court, Suite 300, Kennesaw, GA 30144, USA

2 Akrimax Pharmaceuticals, LLC, Cranford, NJ, USA

3 LT Associates Ltd, Paris, France

4 Department of Neuroscience, University of Oxford, Oxford, UK

5 Arnold Sterman, MD, LLC, Morristown, NJ, USA situations that were more likely attributable to treatment initiation than inadequate therapeutic effect. Trained nurses extracted data from patient charts and electronic medical record systems for review. We analyzed the cost of resources consumed by the frequency of levothyroxine dose changes over 24 months: 0 dose changes (no dose adjustment group); one dose change, two dose changes, three or more dose changes ( $\geq 1$ dose adjustment group).

Results The study included 454 patients. Overall estimated resource utilization was higher per patient in the $\geq 1$ dose adjustment group (US\$5824) vs. the no dose adjustment group (US\$3166) during the 24-month study period. When direct and indirect costs were combined, overall costs of care were greatest in patients requiring three or more dose adjustments (US\$8220/patient). Patients in this cohort incurred 2.5-fold greater total costs compared with patients requiring no dose adjustments (US\$8220 vs. US\$3166). Among the 58 patients in the group requiring three or more dose adjustments, mean direct medical costs were significantly higher than in the patients requiring no dose adjustments (US\$6387 vs. US\$2182). Patients with at least one dose adjustment experienced a $40.3 \%$ increase in lost productivity vs. patients who had no dose adjustments (US\$1381 vs. US\$984). Loss of productivity was highest among patients with three or more levothyroxine dose adjustments. Among this cohort, there was an $86.4 \%$ increase in lost productivity vs. patients who had no levothyroxine dose adjustments (US\$1833 vs. US\$984).

Conclusions Patients experiencing multiple levothyroxine dose adjustments were shown to consume more healthcare resources, resulting in higher costs than those who required no dose adjustments. Each care episode contributed to lost time and wages with total estimated lost productivity escalating with increasing levothyroxine dose adjustments over a 24-month period. 


\section{Key Points}

CONTROL HE study quantifies for the first time the economic burden resulting from levothyroxine dose adjustments.

There were significant differences found in resource consumption across the four dose adjustment cohorts for laboratory testing, thyroid medications, general physician and specialist office visits, and emergency department visits/hospitalizations.

When both direct and indirect costs of care were considered, significant and escalating differences in total costs per patient were observed across the four dose adjustment groups.

Given the multiple reasons for titrating levothyroxine, increased awareness of the economic consequences of such therapy changes may offer an effective tool to educate healthcare providers and improve outcomes for patients with hypothyroidism.

\section{Introduction}

Hypothyroidism is a common endocrine disorder resulting from deficiency of thyroid hormone. It is typically a primary process in which the thyroid gland is unable to produce sufficient amounts of thyroid hormone [1]. Hypothyroidism may be either subclinical or overt. According to treatment guidelines issued by the American Thyroid Association and the American Academy of Clinical Endocrinology, subclinical hypothyroidism is characterized by a serum thyroid level (as determined by a thyroid-stimulating hormone [TSH] test) above the upper reference limit of normal in combination with a normal level of free thyroxine. Both must occur in the absence of ongoing severe illness. An elevated TSH, usually above $10 \mathrm{mIU} / \mathrm{L}$, in combination with subnormal thyroxine characterizes overt hypothyroidism [2]. Subclinical hypothyroidism has been reported to occur with a prevalence of $4.3 \%$ in USA; overt hypothyroidism is reported to occur with a prevalence of $0.3 \%$ [3]. Hypothyroidism is thought to be one of the most frequently encountered disorders in clinical practice.

In general, hypothyroidism can be adequately treated with a consistent daily dose of levothyroxine. Many patients, however, require dose adjustments during the course of levothyroxine therapy [4-9]. Common factors that can necessitate levothyroxine dose adjustments include lack of medication persistence, changes in levothyroxine formulation, dosage errors, concomitant medical conditions/medications, body mass changes, and dietary habits. Levothyroxine is a drug with a narrow therapeutic index and its absorption is dependent on gastric $\mathrm{pH}$. Some gastrointestinal (GI) conditions and their treatments can contribute to suboptimal levothyroxine performance by altering gastric acidity and thereby reducing the bioavailability of levothyroxine [1, 10-31].

The need for levothyroxine dose adjustment is frequent in clinical practice. Despite this, the economic impact of levothyroxine dose adjustments has not previously been determined.

\section{Study Objectives and Goals}

The primary objective of our study was to measure the effect of levothyroxine dose adjustments on the associated utilization of healthcare resources, including direct and indirect costs. We analyzed the cost of resources consumed, stratified by the number of levothyroxine dose changes over 24 months: 0 dose changes; one dose change, two dose changes, and three or more dose changes. In certain analyses, the one dose change, two dose changes, and three or more dose changes cohorts were combined into a one or more dose adjustment group (" $\geq 1$ dose adjustment group") and compared with the 0 dose change group ("no dose adjustment group"). A secondary goal was to identify any differences in patient characteristics that may be associated with levothyroxine dose adjustments.

\section{Methods}

\subsection{Sample Size Calculation}

It was determined that a sample size of 454 would provide $80 \%$ power to detect a difference of $25 \%$ in the mean treatment cost of patients with, vs. without, one or more dose adjustments in a 24-month period. Statistical significance was evaluated at a two-sided $\alpha$-level of 0.05 .

\subsection{Selection of Patients and Inclusion/Exclusion Criteria}

A retrospective medical chart review was conducted among randomly selected patients of healthcare providers selected from a pool of primary care practices (PCPs) who are part of several health systems and who treat a high volume of hypothyroid patients. Health systems that were identified for the study represent several different US regions and include the states of California, Tennessee, and Pennsylvania. Participating health systems identified 
medical records via electronic record compilation that met the study's inclusion criteria. Access to those records was provided for review, with patient names and records blinded to the sponsor. A total of 454 records were randomly selected, 227 per group. Following an institutional review board-approved study protocol, trained nurses extracted data from patient charts and electronic medical record systems for further review.

Patients in each study group were requested to meet the following inclusion criteria: age $>18$ years; diagnosis of hypothyroidism between 31 October 2012 and 30 April 2013; and taking levothyroxine for the treatment of hypothyroidism with the specified number of dosage adjustments ( $\geq 1$ or 0 ) in the 2 -year period between 1 May 12013 and 30 April 2015. Patients in each group were excluded from participation in the study if they were current or past users of Tirosint ${ }^{\circledR}$ (levothyroxine sodium) gel caps or pregnant.

\subsection{Data Source, Data Quality, Selection of Sites}

\subsubsection{Data Source}

Patient screening was accomplished through the use of electronic medical records from partner-identified sites. An initial chart screen was conducted by nurses trained to identify eligible medical records based on study criteria. Once eligible records were identified, a manual chart review was conducted to compile data necessary to conduct analyses.

\subsubsection{Data Quality}

Completed data collection forms were reviewed by a nurse manager for completeness and accuracy. Forms were reviewed, verified, and committed to a final extraction database. The verification process included logical-range checks. A final review of the database for consistency and accuracy was also conducted.

\subsubsection{Selection and Number of Sites}

The study population was representative of 15 PCPs that were identified by the participating healthcare providers as having a high volume of patients with hypothyroidism.

\subsection{Data Collection}

\subsubsection{Medical Record Selection and Sampling}

A total of 454 records were selected for manual chart review. These were sourced from the 15 study sites.
Appendix 1 in the Supplementary Material lists the information that was collected from medical charts.

\subsubsection{Medical Chart Extraction, Review, and Verification of Data Quality}

Trained nurses underwent an inter-rater reliability assessment with a nurse manager and were required to score at least $90 \%$. Forms were reviewed, verified, and committed to a final extraction database. The verification process included logical-range checks.

\subsection{Statistical Analysis}

\subsubsection{Study Endpoints/Outcomes, Analysis Set}

Study endpoints/outcomes were as follows:

- Direct medical costs per patient including the costs of physician office visits, referrals to specialists, laboratory tests, medication costs, emergency department (ED) visits, and hospitalization;

- Indirect medical costs per patient including the cost of lost productivity resulting from the above;

- Characteristics of the study population including demographics, comorbidities, concomitant medications, and other factors that may negatively affect levothyroxine therapy and contribute to the need for dose changes.

All patients meeting the study inclusion criteria were analyzed in the full analysis set.

\subsection{Method of Analysis}

Data were managed in Microsoft Excel and all analyses performed using SAS Version 9.2 software (Cary, NC, USA). Summaries for the two study groups, and for all patients as a whole, are presented. Demographic data, including age, sex, race, and ethnicity are presented in Table 2. Analysis of mean direct and indirect costs, with 95\% confidence intervals, are provided in subsequent tables. For comparison of mean direct and indirect costs between the no dose adjustment group and the $\geq 1$ dose adjustment group, a $t$ test was used. For comparison of mean direct and indirect costs between the no dose adjustment, one-dose adjustment, two-dose adjustments, and three or more dose adjustments groups, a one-factor analysis of variance (ANOVA) was used. A Tukey posthoc multiple comparison test was used, when appropriate, following significant ANOVA results. Significance was evaluated at a two-sided $\alpha$-level of 0.05 unless otherwise stated. 


\subsection{Study Ethics/Institutional Review Board Information}

The study protocol was reviewed and approved by the Sterling Institutional Review Board, Atlanta, GA, USA. To ensure the research was in compliance with Health Insurance and Accountability Act of 1996 (HIPAA) legislation, a waiver of authorization was obtained according to 45 CFR 164.512 (i)(1)(i), which allowed the collection of protected health information without the authorization of the research participants for research purposes.

All collected patient information was de-identified. To assure patient confidentiality, a unique identifier, patient name, and date of birth were not extracted. Only the unique identifier was stored in the excel sheets and analyzed. A business associate/confidentiality agreement was executed with each participating site.

In compliance with federal regulations, direct access to patient information was permitted by authorized personnel to review patients' original medical records for verification of study-related activities and data. Direct access to patient information included examining, analyzing, verifying, and reproducing any records and reports that were important to the evaluation of the study.

\subsection{Methods/Cost Variations}

The overall costs of care for patients included estimates of average direct medical costs paid to providers, such as investigations (i.e., pathology and imaging), monitoring (e.g., TSH tests, electrocardiograms), PCP visits, and specialist visits. A direct cost for medication was associated with each levothyroxine formulation dosage for the period of time the patient was on that drug during the study (in 30-day increments). Hospitalizations and ED visits were analyzed if relevant to thyroid disease and levothyroxine. All other resources used, including PCP visits, specialist visits, and laboratory results, were captured as a whole for the entire cohort. Dose changes that occurred with patients who were recently started on levothyroxine therapy ( $<6$ months) were excluded to avoid situations that were more likely attributable to treatment initiation than inadequate therapeutic effect. For ED visits and hospitalization, only patients whose symptoms upon hospital presentation that were consistent with hyperthyroidism (e.g., tachycardia, tremors, anxiety) and who experienced a post-discharge reduction in levothyroxine dose within 3 weeks of the documented ED visit or hospitalization were included. Additionally, a subgroup analysis of patients with GI disorders was conducted, based on recently published data demonstrating the negative effect that some GI medications can have on levothyroxine efficacy, which may lead to dose adjustments. All costs were estimated per patient over a 24-month period based on actual utilization of resources extracted from charts. The methods used to determine cost per care event are summarized in Table 1.

\section{Results}

\subsection{Demographics}

Of the 454 distinct patients whose medical records were examined, all were receiving levothyroxine monotherapy (i.e., not combined with triiodothyronine [T3] treatment). The mean ages of patients in the no dose adjustment and $\geq 1$ dose adjustment groups were comparable: 57 years for the no dose adjustment group and 58 years for the $\geq 1$ dose adjustment group. It is important to note that the prevalence of hypothyroidism peaks between the ages of 50 and 74 years [41], and across both groups in the present study, 248 patients $(54.6 \%)$ were between the ages of 50 and 74 years, inclusive. Caucasian female individuals $(80.2 \%)$ represented the most common patient type. Table 2 describes the study population.

\subsection{Overall Costs Per Patient}

Overall estimated resource utilization was higher per patient in the $\geq 1$ dose adjustment group (US\$5824) vs. the no dose adjustment group (US\$3166) during the 24-month study period (Table 3 ). The principal components of the difference between the two groups include the costs for physician office visits, laboratory tests, ED visits/hospitalizations, and lost productivity. The cost per patient for direct costs (laboratory tests, medication, office visits, and ED visits/hospitalizations) accounted for $82.4 \%$ (US\$2190/ US\$2658) of the total estimated difference in cost between the no dose adjustment and $\geq 1$ dose adjustment groups; $17.6 \%$ of the difference was in indirect costs (lost productivity).

\subsection{Direct Medical Costs by Dose Stratification}

Results show that the direct cost of care escalated with the increasing number of levothyroxine dose adjustments. Costs for patients who received one dose adjustment were US\$1557 greater than for those with no dose adjustment (US\$3739 vs. US\$2182, as indicated in Table 4). As can be seen in Table 4, there were significant differences found in resource consumption across the four dose adjustment cohorts for laboratory work, thyroid medications, specialist costs, office visits, and ED visits/hospitalization. The apparent decrease in direct medical costs among patients with two dose adjustments vs. one dose adjustment was noted. This was driven by lower costs for thyroid 


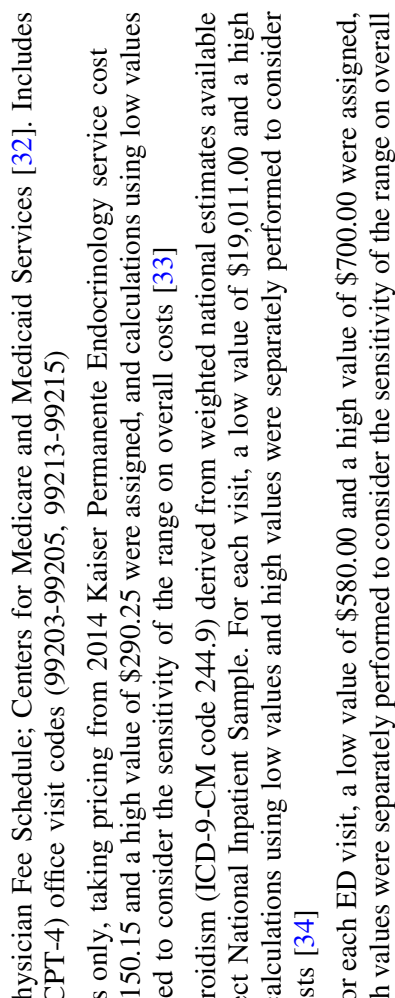

公它

它面

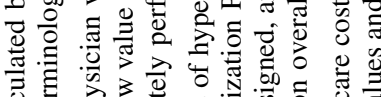

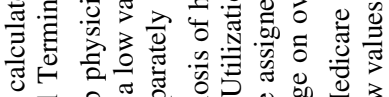

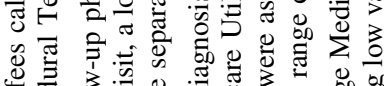

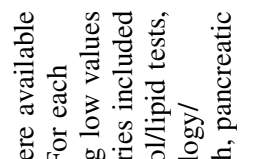

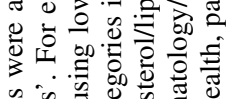

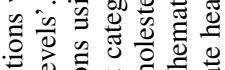

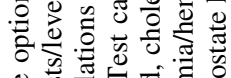

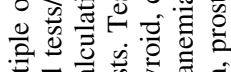

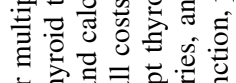

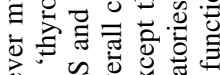

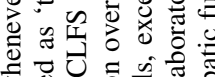

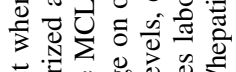

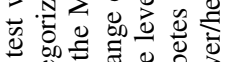

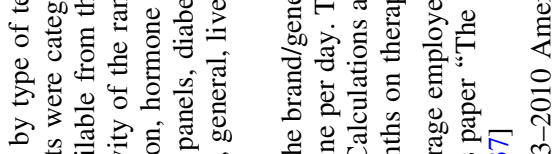

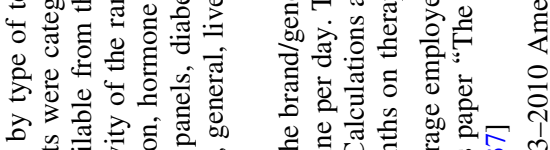

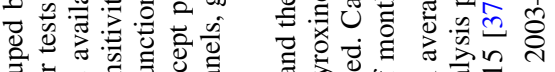

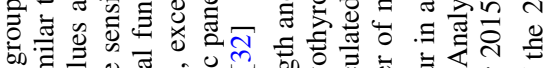

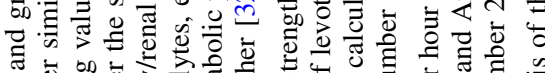

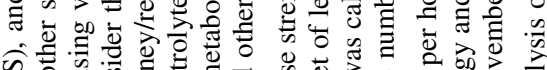

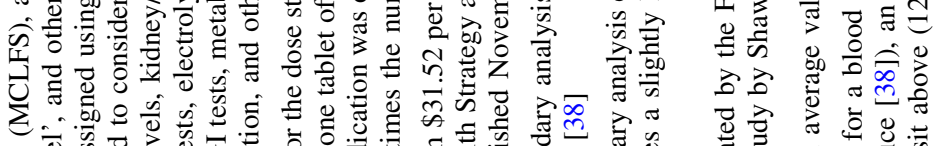

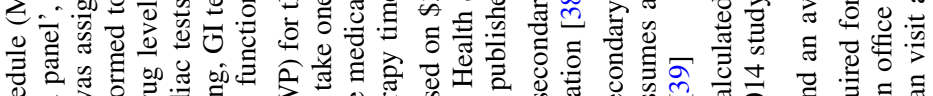

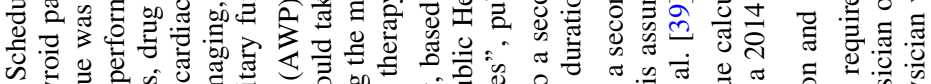

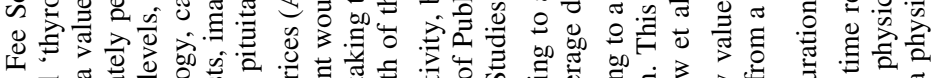

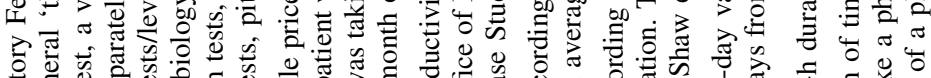

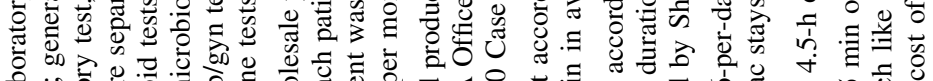

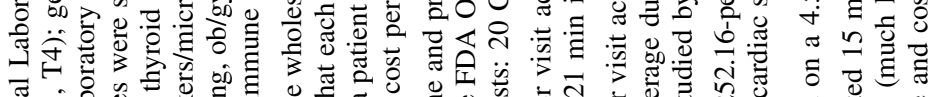
矢递

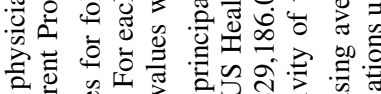

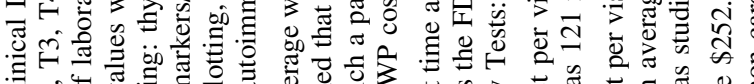

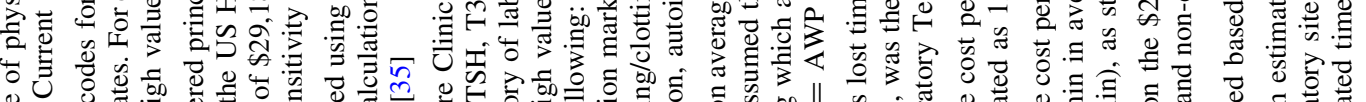

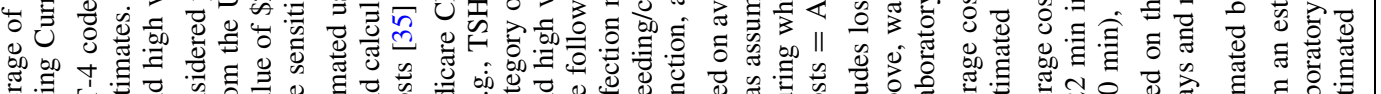

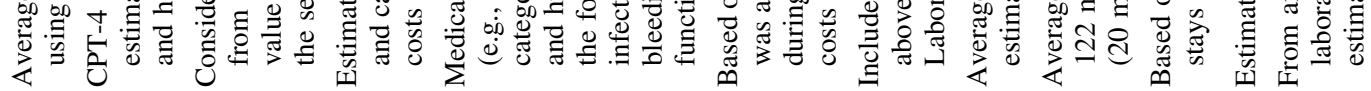
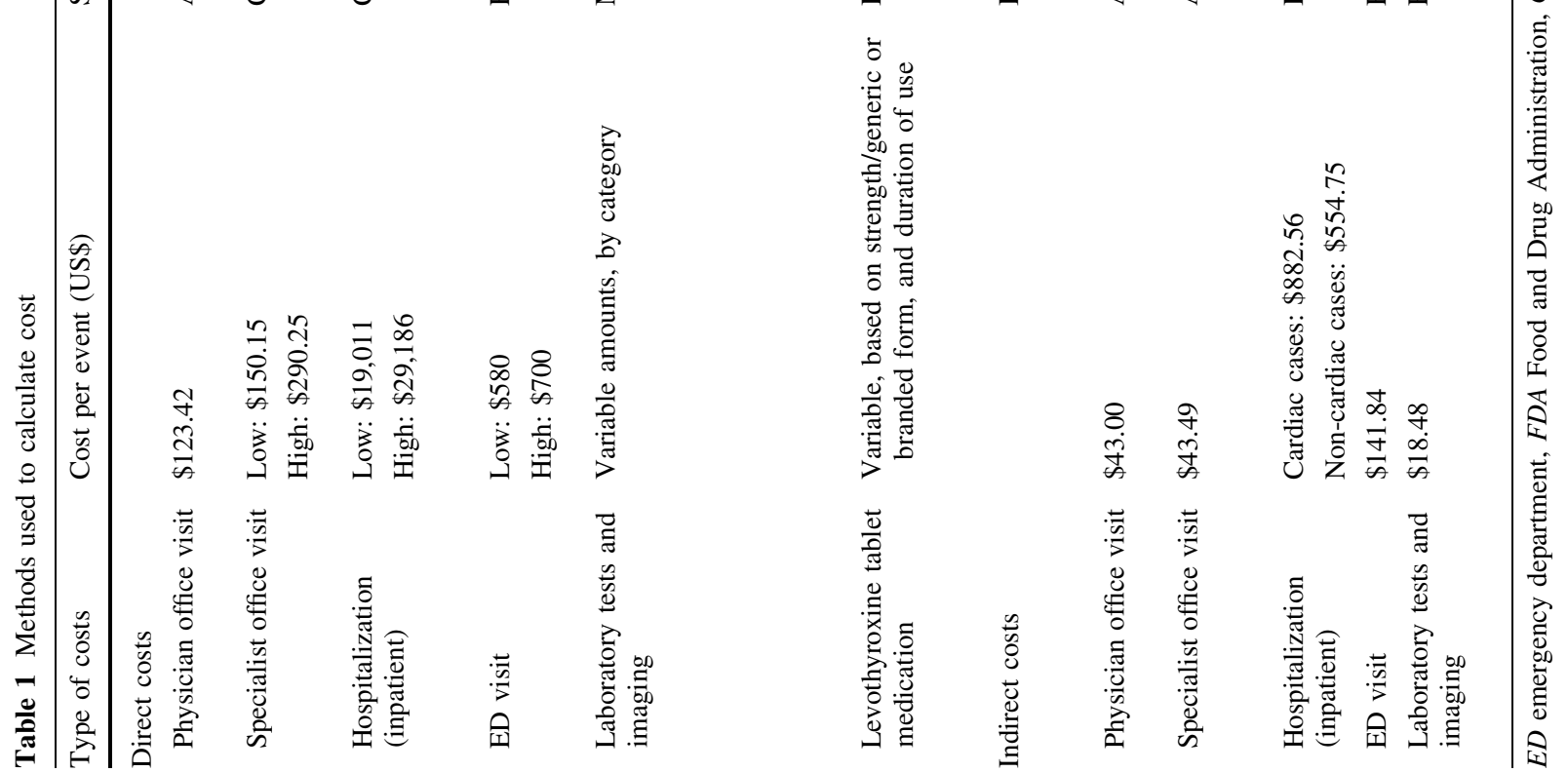
Table 2 Patient demographics

\begin{tabular}{lll}
\hline Variable & $\begin{array}{l}\text { No dose adjustment } \\
\text { group }(n=227)\end{array}$ & $\begin{array}{l}\geq 1 \text { dose adjustment } \\
\text { group }(n=227)\end{array}$ \\
\hline Age, years & & 58 \\
Mean & 57 & 15 \\
Standard deviation & 16 & 20 \\
Minimum & 18 & 97 \\
Maximum & 93 & \\
Sex, \% $(n)$ & & $84(190)$ \\
Female & $77(174)$ & $16(37)$ \\
Male & $23(53)$ & \\
Race, \% (n) & & $85(192)$ \\
White/Caucasian & $75(170)$ & $4(9)$ \\
African American & $4(10)$ & $1(1)$ \\
Asian & $0(0)$ & $0(0)$ \\
Other & $3(6)$ & $10(25)$ \\
Unknown & $18(41)$ & \\
Patient ethnicity, \% $(n)$ & & $2(5)$ \\
Hispanic/Latino & $4(8)$ & $58(132)$ \\
Not Hispanic/Latino & $62(140)$ & $40(90)$ \\
Unknown & $35(79)$ & \\
\hline
\end{tabular}

medication, office visits, ED visits, and hospitalizations. The reasons for these differences could not be discerned from the data available. However, the difference in total cost between the two-dose adjustment and one-dose adjustment groups was not statistically significant.

As displayed in Fig. 1 in the Supplementary Material, total direct medical costs per patient were significantly greater in patients requiring three or more dose adjustments, compared with those requiring no dose adjustments (US\$6837 vs. \$2182; $p<0.05$ ).

\subsection{Lost Productivity}

As displayed in Table 5, each care episode contributed to lost time and wages with total estimated lost productivity escalating with increasing levothyroxine dose adjustments. Patients with multiple dose adjustments had greater total lost productivity cost when ED visits and hospitalizations were considered. Patients with one dose adjustment experienced a $40.3 \%$ increase in lost productivity cost vs. patients who had no dose adjustments (US\$1381 vs. US\$984).

Patients with one or more dose adjustments had an increased loss of productivity cost of US $\$ 469$ per patient vs. patients who had no dose adjustment. Loss of productivity cost was highest among patients with three or more levothyroxine dose adjustments. Among this cohort, there was an $86.4 \%$ increase in lost productivity cost vs. patients who had no levothyroxine dose adjustments (US\$1833 vs. US\$984).

\subsection{Total Cost of Care}

When both direct and indirect costs of care were considered, a significant and escalating difference in total costs per patient was observed across the four dose adjustment groups $(p<0.05)$ (Table 6). When direct and indirect costs were combined, overall costs of care were greatest in subjects requiring three or more dose adjustments (US\$8220). Patients in this cohort experienced a $160 \%$ increase in total cost per patient compared with patients requiring no dose adjustments during the 24-month study period (US\$8220 vs. US\$3166; $p<0.05$ ) (Fig. 2 in the Supplementary Material).

Table 3 Total estimated costs (US\$) per patient: no dose adjustment vs. $\geq 1$ dose adjustment groups

\begin{tabular}{|c|c|c|c|c|c|c|}
\hline \multirow[t]{2}{*}{ Component } & \multicolumn{2}{|c|}{ No dose adjustment group, $n=227$} & \multicolumn{2}{|c|}{$\geq 1$ dose adjustment group, $n=227$} & \multicolumn{2}{|l|}{ Difference } \\
\hline & Cost per patient $(95 \% \mathrm{CI})$ & $\%$ of total & Cost per patient $(95 \% \mathrm{CI})$ & $\%$ of total & Cost per patient & $\%$ Change $^{\mathrm{a}}$ \\
\hline Laboratory tests & $\$ 262(\$ 220-\$ 303)$ & 8.3 & $\$ 467(\$ 421-\$ 513)$ & 8.0 & $\$ 206$ & $+78.6^{*}$ \\
\hline Thyroid medication & $\$ 546(\$ 515-\$ 577)$ & 17.2 & $\$ 663(\$ 591-\$ 735)$ & 11.4 & $\$ 117$ & $+21.5^{*}$ \\
\hline Specialist visits & $\$ 156(\$ 123-\$ 188)$ & 4.9 & $\$ 169(\$ 135-\$ 203)$ & 2.9 & $\$ 13$ & $+8.2 *$ \\
\hline Office visits & $\$ 1218(\$ 1020-\$ 1415)$ & 38.5 & $\$ 1850(\$ 1635-\$ 2066)$ & 31.8 & $\$ 632$ & $+51.9 *$ \\
\hline ED visits + hospitalizations & $\$ 0$ & 0.0 & $\$ 1222(\$ 452-\$ 1992)$ & 21.0 & $\$ 1222$ & - \\
\hline Subtotal & $\$ 2182(\$ 1955-\$ 2408)$ & 68.9 & $\$ 4372(\$ 3520-\$ 5223)$ & 75.1 & $\$ 2190$ & $+100.4^{*}$ \\
\hline Estimated lost productivity & $\$ 984(\$ 832-\$ 1135)$ & 31.1 & $\$ 1452(\$ 1295-\$ 1609)$ & 24.9 & $\$ 468$ & $+47.6^{*}$ \\
\hline Estimated total costs & $\$ 3166(\$ 2801-\$ 3529)$ & 100 & $\$ 5824(\$ 4863-\$ 6784)$ & 100 & $\$ 2658$ & $+84.0 *$ \\
\hline
\end{tabular}

$C I$ confidence interval, $E D$ emergency department

* Significant difference between groups $(p<0.05)$

a $\%$ Change $=(\geq 1$ dose adjustment group - no dose adjustment group $) /$ no dose adjustment group. A $t$ test was used to compare costs between groups 


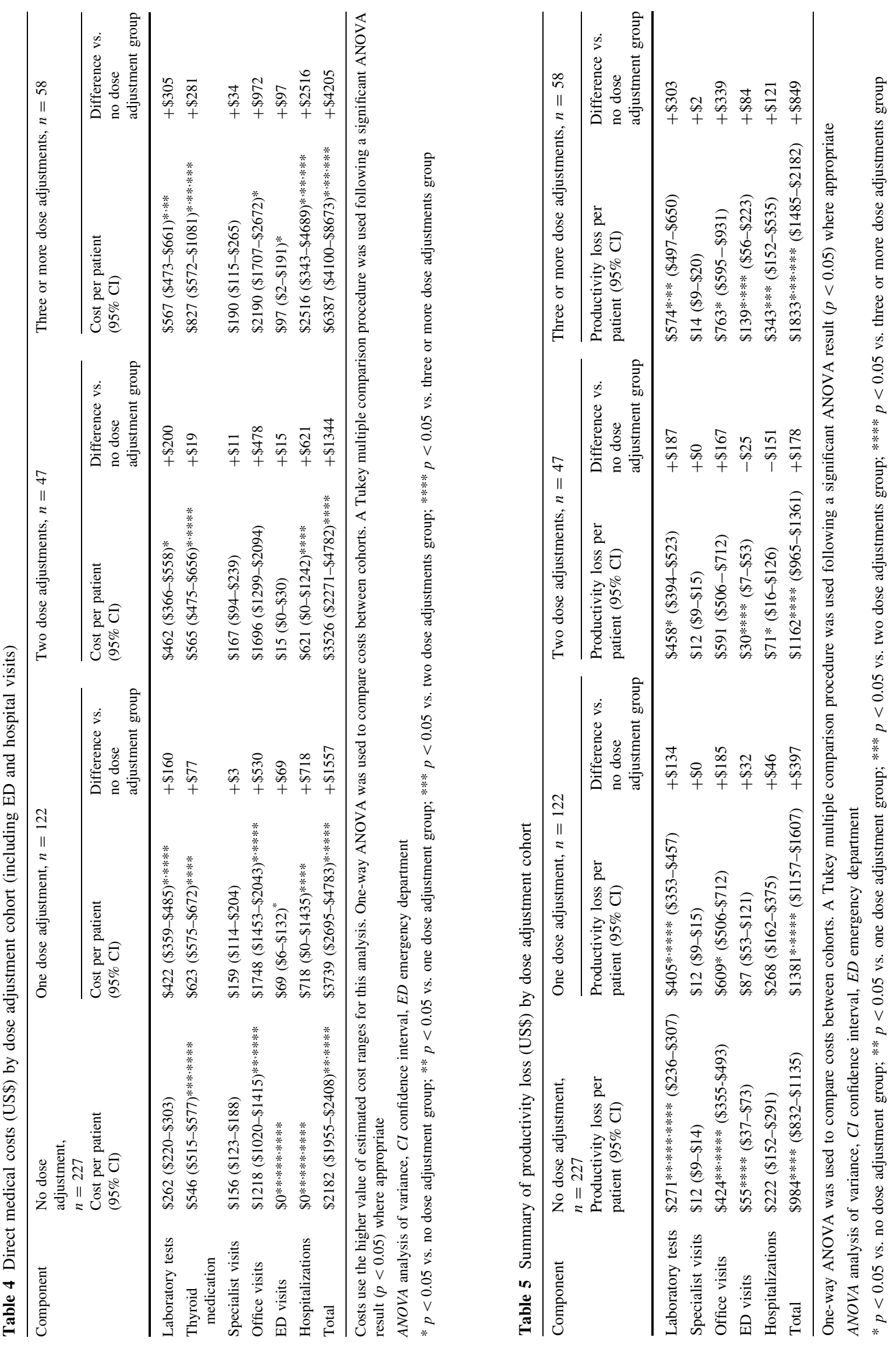


Table 7 Mean TSH levels by patient group

\begin{tabular}{lcll}
\hline & $\begin{array}{l}\text { TSH levels } \\
\text { in no dose } \\
\text { adjustment group } \\
(\mathrm{U} / \mathrm{mL})\end{array}$ & $\begin{array}{l}\text { TSH levels } \\
\text { in } \geq 1 \text { dose } \\
\text { adjustment group } \\
(\mathrm{U} / \mathrm{mL})\end{array}$ & $p$ value \\
\hline$n$ & 165 & 215 & $<0.001^{\mathrm{a}}$ \\
Mean & 2.57 & 5.07 & $<0.001^{\mathrm{b}}$ \\
$\mathrm{SD}$ & 2.51 & 11.04 & \\
Minimum & 0.06 & 00.1 & \\
Maximum & 15.9 & 100 &
\end{tabular}

TSH thyroid-stimulating hormone, $S D$ standard deviation

${ }^{a} p$ value calculated using chi-square to compare proportion of patients in each group who had TSH testing (i.e., 165/227 vs. 215/227)

b $p$ value calculated using $t$ test to compare mean TSH levels between groups

\subsection{Other Findings}

A secondary objective of CONTROL HE was to examine patient characteristics that may help to explain the differences in levothyroxine dosing requirements. Among patients requiring levothyroxine dose changes, the following findings were observed:

- Higher TSH levels Patients requiring thyroid medication dose adjustment had significantly higher mean TSH levels compared with those who did not require levothyroxine dose adjustments $(5.07$ vs. $2.57 \mathrm{U} / \mathrm{mL}$, $p<0.001$ ) (Table 7). TSH testing was performed more often during the 24-month study period among patients requiring dose adjustment than patients without dose adjustment (215 [84\%] vs. 165 [73\%]; $p<0.001)$.

- Presence of GI conditions Patients requiring thyroid medication dose adjustments were more likely to experience GI disorders than patients without dose adjustment (103 [45\%] vs. 83 [36\%]; $p>0.05$ ) [Fig. 3 in the Supplementary Material]. Common GI disorders observed were gastroesophageal reflux disease, irritable bowel syndrome, and lactose intolerance.

CONTROL HE adds to the body of clinical literature documenting the adverse effect that some GI conditions and their treatments can have on the performance of levothyroxine. More than $45 \%$ of patients requiring levothyroxine dose adjustments (103/227) were being treated for GI disease. These patients were more likely to incur additional treatment costs (Table 8). Among patients with GI conditions that required three or more levothyroxine dose adjustments, an additional US\$5046 of direct medical costs were incurred during the 24-month study period when compared with patients with no dose adjustment. 


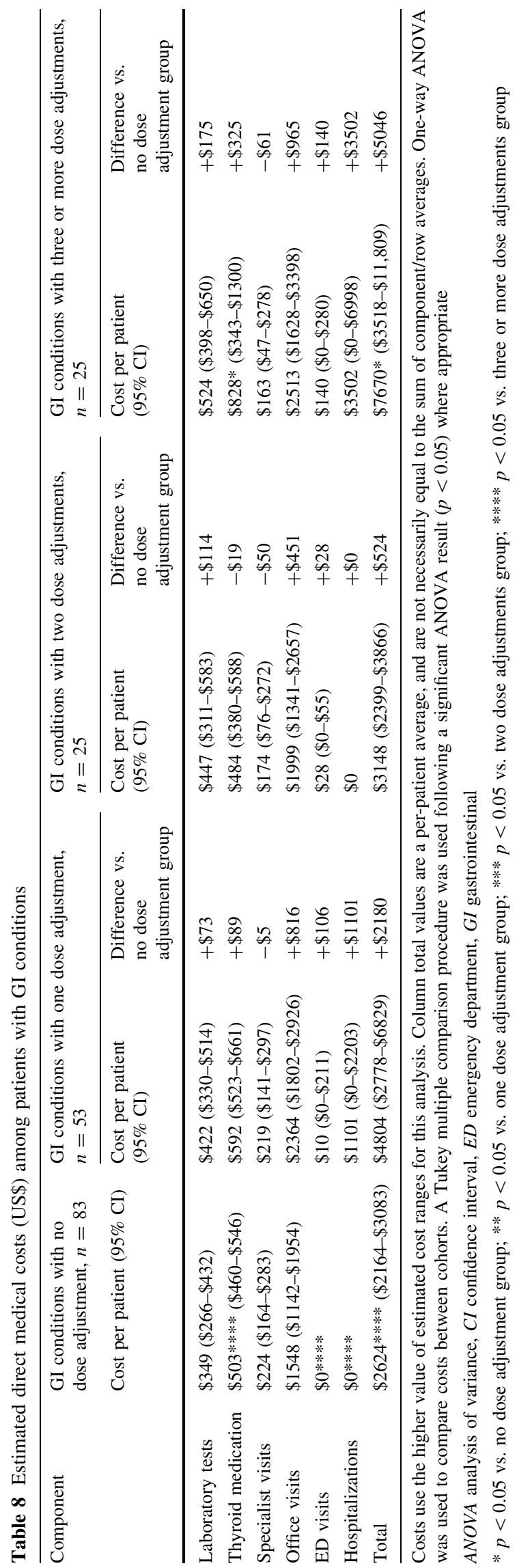

- Use of concomitant medication or dietary supplements Significantly more patients needing thyroid medication dose adjustments were also taking drugs/supplements that might interfere with levothyroxine absorption: (196 [86\%] vs. 169 [75\%]; $p=0.002$ ) (Fig. 4 in the Supplementary Material). Common drugs/supplements used by patients requiring levothyroxine dose adjustments included proton pump inhibitors (PPIs, by prescription and over the counter), histamine 2 receptor antagonists, and calcium or iron supplements.

\section{Discussion}

Thyroid hormone replacement therapy with oral levothyroxine has been considered the "gold standard" for treating hypothyroidism for over 60 years. It is one of the most frequently used medications in USA, with over 115 million prescriptions dispensed in 2013 [41]. Most cases of hypothyroidism can be adequately treated with a consistent daily dose of levothyroxine. However, as noted above, many patients require levothyroxine dose adjustments to maintain desired TSH levels and to adequately control hypothyroid symptoms [4-9]. Factors that can necessitate levothyroxine dose adjustment include lack of medication persistence, changes in body mass, dosage errors, GI comorbidities, concomitantly used medication, diet, and changes in levothyroxine formulations.

As mentioned above, the absorption of levothyroxine can be limited, and its effectiveness adversely affected, by a variety of diseases of the GI tract, including inflammatory bowel disease, atrophic gastritis, celiac disease, Helicobacter pylori infection, gastroesophageal reflux disease, lactose intolerance, and gastroparesis, among others. Accordingly, the presence of these conditions may adversely affect levothyroxine dose requirements [1, 10-21].

Additional factors that have been shown to affect levothyroxine absorption and effectiveness-some of which may be related to the diseases of the GI tract noted above-include excess body weight, poor compliance with therapy, diet, gastric bypass surgery (reduced or delayed absorption), and use of certain nutritional supplements, vitamins, and medications, such as PPIs, histamine receptor blockers, cholestyramine, and motilitymodifying agents $[1,11,12,16-19]$. As also indicated by the results of the present study, the above factors have been shown to lead to unnecessary consumption of healthcare resources in the form of increased physician and pharmacy visits, laboratory costs, and medication adjustments [22-31]. 
Table 9 Per-patient cost comparison (US\$) of selected chronic disease categories/drugs per year. Adapted from Hallert 2014; Owens 2014; Sun 2014; Rachana 2014 [44-47]

\begin{tabular}{lccc}
\hline Disease & $\begin{array}{l}\text { Estimated direct } \\
\text { costs }\end{array}$ & $\begin{array}{l}\text { Estimated lost } \\
\text { productivity costs }\end{array}$ & $\begin{array}{l}\text { Estimated total } \\
\text { costs }\end{array}$ \\
\hline Hypothyroidism, no dose changes $^{\mathrm{a}}$ & $\$ 1091$ & $\$ 492$ & $\$ 1583$ \\
Hypothyroidism, three or more dose changes $^{\mathrm{a}}$ & $\$ 3194$ & $\$ 916$ & $\$ 4110$ \\
Rheumatoid arthritis [44, 45] $_{\text {Fibromyalgia [46] }}$ & $\$ 5720$ & $\$ 5822$ & $\$ 11,542$ \\
Hypertension [47] & $\$ 10,312$ & $\$ 4950$ & $\$ 15,262$ \\
\hline
\end{tabular}

${ }^{a}$ Estimated levothyroxine annual costs are $50 \%$ of 24 -month study period costs (Table 6)
In CONTROL HE, patients who were being treated for certain GI diseases and experienced three or more levothyroxine dose adjustments were more likely to incur additional treatment costs. This finding is consistent with recently published data demonstrating the negative effect that some GI medications can have on levothyroxine effectiveness. In the TEARS Study $(n=10,999)$, Irving et al. demonstrated that patients taking PPIs experienced a $7.89 \%$ increase in serum TSH levels $(p=0.01)$ when taking these medications concomitantly with levothyroxine [42].

Levothyroxine dose adjustments are common in clinical practice. In the recently completed CONTROL Surveillance Project conducted among 1000 levothyroxine-treated patients (925 on levothyroxine monotherapy), over $31 \%$ reported that they had experienced one or more levothyroxine dose changes in the prior 12 months. Among patients reporting dose changes, the majority (60\%) stated that they had experienced one such change; $40 \%$ reported two or more such changes. It is important to note than $94 \%$ of patients in CONTROL Surveillance reported being treated with levothyroxine for more than 10 years [43].

In CONTROL HE, almost half of all patients experiencing levothyroxine dose changes $(46.3 \%)$ received two or more dose changes. These led to escalating costs of care, both direct medical costs and the costs of lost productivity, during the 24-month study period (Table 8). Using data from CONTROL HE, the total cost of levothyroxine for 1000 patients treated is estimated to range from US $\$ 3,165,210$ (no levothyroxine dose adjustments) to US $\$ 8,220,540$ (three or more levothyroxine dose adjustments).

Direct medical costs represent the majority of these costs across all study cohorts. Among patients requiring three or more dose adjustments, direct medical costs represent $78 \%$ of total estimated costs (US\$6,386,830/ US $\$ 8,220,540$ ) for 1000 treated patients, based on the study results above (Table 1 in the Supplementary Material). The cost of lost work productivity is estimated to range from US $\$ 983,680$ per 1000 treated patients for those experiencing no levothyroxine dose changes to US $\$ 1,833,710$ per 1000 treated patients for those requiring three or more levothyroxine dose adjustments.

While the estimated costs of levothyroxine therapy may seem high, they appear modest when compared with other common chronic conditions (Table 9). Among patients in CONTROL HE who experienced no levothyroxine dose adjustments, the total cost of care is estimated at US\$1583 per annum. The primary contributors to this include laboratory testing, office visits, drugs to treat hypothyroidism, and lost work productivity. This picture changes when frequent levothyroxine dose changes are required. Among patients requiring multiple levothyroxine dose changes, the estimated cost of managing hypothyroidism escalates to US $\$ 4110$ per annum, still less than half of the estimated annual cost to treat rheumatoid arthritis or hypertension.

CONTROL HE indicates that there may be significant costs associated with excessive exposure to levothyroxine. As mentioned previously, only ED visits or hospital stays in which presenting symptoms were consistent with hyperthyroidism, and which were associated with a reduction in levothyroxine dose post discharge, were included in the analysis. In CONTROL HE, the direct medical costs per patient associated with ED visits and hospitalizations ranged from US\$0 in the no dose change group to US\$2613 in the three or more dose changes group (Table 4).

Patients in the two levothyroxine dose change group experienced a $24 \%$ reduction in costs associated with ED visits and hospitalizations compared with patients in the one dose change group (US\$636 vs. US\$787; $p=0.8320$ ). This explains most of the difference in total direct medical costs observed between these two groups discussed previously (US $\$ 3739$ for the one dose change group vs. $\$ 3526$ for the two dose change group ( $p=0.7923)$.

The results of CONTROL HE relative to ED and hospitalization costs are not implausible. Data from five different studies have shown that excessive exposure to levothyroxine is common, ranging from 14 to $22 \%$ of all patients [3, 48-51]. Furthermore, while many patients with excessive levothyroxine exposure may not require ED or 
hospital care, the economic impact of excessive levothyroxine exposure remains an open question that should be further investigated with an adequately powered study.

\subsection{Study Limitations}

Even though adequately powered, the size of the CONTROL HE Study (454 patients) limits its ability to offer a complete picture of the levothyroxine-taking population. The study represents a cohort of patients treated for hypothyroidism in ambulatory settings in USA, from physicians who agreed to participate in a medical record review. The population studied may not be representative of the overall levothyroxine-taking population. While the prevalence of factors know to interfere with levothyroxine therapy has been noted, the study does not provide exact reasons why some patients require a higher number of levothyroxine dose adjustments compared with a matched cohort that differed only in the absence of dose changes. Finally, while we derived estimates for both direct medical and lost productivity costs from well-documented sources, these cost estimates may vary by geography and health systems. Despite a large variability of direct and indirect costs depending on the country examined, the lack of an individually tailored levothyroxine treatment is always expensive. Therefore, CONTROL HE is neither simply hypothesis generating, nor conclusive, but certainly provides new and essential information to understand the puzzle of levothyroxine treatment in clinical practice today.

\section{Conclusions}

Based on a literature review, we believe that CONTROL $\mathrm{HE}$ is the only known attempt to date to quantify the economic burden resulting from levothyroxine dose adjustments that occur frequently in clinical practice. In CONTROL HE, patients experiencing multiple levothyroxine dose adjustments were shown to consume more healthcare resources than those who required no dose adjustments. Common factors associated with the need for levothyroxine dose adjustments (the presence of GI disease, the use of concomitant medication and dietary supplements) have been noted extensively in the clinical literature. CONTROL HE adds to this body of evidence and provides specific estimates of their economic impact, especially the costs of using levothyroxine to treat hypothyroid patients with concomitant GI disease.

The results from CONTROL HE suggest an extensive number of ED visits and hospitalizations may be associated with levothyroxine over-exposure. In CONTROL HE, these episodes were shown to lead to incremental direct medical costs and reduced worker productivity. This finding should be explored in more rigorously designed studies as its implications for healthcare payers and society may be significant.

In summary, CONTROL HE adds to our overall understanding of levothyroxine. Given the multiple reasons for titrating levothyroxine, increased awareness of the economic consequences of such therapy changes may offer an effective tool to educate healthcare providers and improve outcomes for patients with hypothyroidism.

Acknowledgements The sponsor thanks Indegene, Inc. (Kennesaw, GA, USA) for conducting and overseeing all aspects of this study, and Aesculapius Consulting, Inc. (East Brunswick, NJ, USA) for providing editorial assistance in the development of this article. Additionally, we thank Michelle $\mathrm{Vu}$, a PharmD candidate at Mercer University College of Pharmacy, for her medical literature review of direct medical costs of care.

\section{Compliance with Ethical Standards}

Funding This study was funded by Akrimax Pharmaceuticals, LLC (Cranford, NJ, USA). Editorial assistance, supported by Akrimax, was provided by Aesculapius Consulting, Inc. Honoraria and consulting fees were paid by Akrimax, as detailed in the Conflict of Interest section that follows.

Conflict of interest Frank Ernst, Peri Barr, and Riad Elmor are employees of Indegene, Inc., which received a fee for services related to the development and execution of this study, and for the tabulation, analysis, and reporting of its results. Walter Sandulli and Jessica Goldenberg are employees of Akrimax. Arnold Sterman has been a consultant for Akrimax, has contributed to research funded by Akrimax, and received an honorarium for his contributions to evaluating this study and to the development of this manuscript. Lionel Thevasathan has no conflicts of interest to declare. Kevin Vora was employed by Akrimax at the time of this study.

Ethical standards The study protocol was reviewed and approved by Sterling Institutional Review Board, Inc., Atlanta, GA, USA. To ensure the research was in compliance with HIPAA legislation, a waiver of authorization was obtained according to 45 CFR 164.512 (i)(1)(i), which allowed the collection of protected health information without the authorization of the research participants for research purposes.

Informed consent No written consent was obtained from patients (see Ethical Standards above).

Open Access This article is distributed under the terms of the Creative Commons Attribution-NonCommercial 4.0 International License (http://creativecommons.org/licenses/by-nc/4.0/), which permits any noncommercial use, distribution, and reproduction in any medium, provided you give appropriate credit to the original author(s) and the source, provide a link to the Creative Commons license, and indicate if changes were made.

\section{References}

1. Ward LS. The difficult patient: drug interaction and the influence of concomitant diseases on the treatment of hypothyroidism. Arq Brasil Endocrinol Metab. 2010;54:435-42. 
2. Garber JR, Cobin RH, Gharib H, et al. Clinical practice guidelines for hypothyroidism in adults: cosponsored by the American Association of Clinical Endocrinologists and the American Thyroid Association. Thyroid. 2012;22:1200-35.

3. Hollowell JG, Staehling NW, Flanders WD, et al. Serum TSH, T4, and thyroid antibodies in the United States population (1988 to 1994): National Health and Nutrition Examination Survey (NHANES III). J Clin Endocrinol Metab. 2002;87:489-99.

4. Dar RA, Chowdri NA, Parray FQ, Wani SH. An unusual case of Hashimoto's thyroiditis with four lobed thyroid gland. N Am J Med Sci. 2012;4:151-3.

5. Yaturu S, Fontinot J, Rowland T. Mixed medullary thyroid cancer and follicular cancer. Am J Case Rep. 2011;12:1-4.

6. Robertson HM, Narayanaswamy AK, Pereira O, et al. Factors contributing to high levothyroxine doses in primary hypothyroidism: an interventional audit of a large community database. Thyroid. 2014;24:1765-71.

7. Santini F, Pinchera A, Marsili A, et al. Lean body mass is a major determinant of levothyroxine dosage in the treatment of thyroid diseases. J Clin Endocrinol Metab. 2005;90:124-7.

8. Roos A, Linn-Rasker SP, van Domburg RT, et al. The starting dose of levothyroxine in primary hypothyroidism treatment: a prospective, randomized, double-blind trial. Arch Intern Med. 2005;165:1714-20.

9. Mandel SJ, Brent GA, Larsen PR. Levothyroxine therapy in patients with thyroid disease. Ann Intern Med. 1993;119:492-502.

10. Virili C, Bassotti G, Santaguida MG, et al. Atypical celiac disease as cause of increased need for thyroxine: a systematic study. J Clin Endocrinol Metab. 2012;97:E419-22.

11. Khandelwal D, Tandon N. Overt and subclinical hypothyroidism: who to treat and how. Drugs. 2012;72:17-33.

12. Liwanpo L, Hershman JM. Conditions and drugs interfering with thyroxine absorption. Best Pract Res Clin Endocrinol Metab. 2009;23:781-92.

13. Badillo R, Francis D. Diagnosis and treatment of gastroesophageal reflux disease. World J Gastrointest Pharmacol Ther. 2014;5:105-12.

14. Cohen E, Bolus R, Khanna D, et al. GERD symptoms in the general population: prevalence and severity versus care-seeking patients. Dig Dis Sci. 2014;59:2488-96.

15. Fasano A, Catassi C. Clinical practice: celiac disease. N Engl J Med. 2012;367:2419-26.

16. Miller AD, Smith KM. Medication and nutrient administration considerations after bariatric surgery. Am J Health Syst Pharm. 2006;63:1852-7.

17. Gaitonde DY, Rowley KD, Sweeney LB. Hypothyroidism: an update. Am Fam Physician. 2012;86:244-51.

18. Cellini M, Santaguida MG, Gatto I, et al. Systematic appraisal of lactose intolerance as cause of increased need for oral thyroxine. J Clin Endocrinol Metab. 2014;99:E1454-8.

19. Ianiro G, Mangiola F, Di Rienzo TA, et al. Levothyroxine absorption in health and disease, and new therapeutic perspectives. Eur Rev Med Pharmacol Sci. 2014;18:451-6.

20. Benvenga $\mathrm{S}$. When thyroid hormone replacement is ineffective? Curr Opin Endocrinol Diabetes Obes. 2013;20:467-77.

21. Checchi S, Montanaro A, Pasqui L, et al. L-thyroxine requirement in patients with autoimmune hypothyroidism and parietal cell antibodies. J Clin Endocrinol Metab. 2008;93:465-9.

22. McMillan C, Bradley C, Razvi S, Weaver J. Psychometric evaluation of a new questionnaire measuring treatment satisfaction in hypothyroidism: the ThyTSQ. Value Health. 2006;9:132-9.

23. Quinque EM, Villringer A, Kratzsch J, Karger S. Patient-reported outcomes in adequately treated hypothyroidism: insights from the
German versions of ThyDQoL, ThySRQ and ThyTSQ. Health Qual Life Outcomes. 2013;11:68.

24. Santaguida MG, Virili C, Del Duca SC, et al. Thyroxine softgel capsule in patients with gastric-related T4 malabsorption. Endocrine. 2015;49:51-7.

25. Collins D, Wilcox R, Nathan M, Zubarik R. Celiac disease and hypothyroidism. Am J Med. 2012;125:278-82.

26. Centanni M. Thyroxine treatment: absorption, malabsorption, and novel therapeutic approaches. Endocrine. 2013;43:8-9.

27. Dorval E, Rey JF, Soufflet C, et al. Perspectives on gastroesophageal reflux disease in primary care: the REFLEX study of patient-physician agreement. BMC Gastroenterol. 2011;11:25.

28. Walker JN, Shillo P, Ibbotson V, et al. A thyroxine absorption test followed by weekly thyroxine administration: a method to assess non-adherence to treatment. Eur $\mathrm{J}$ Endocrinol. 2013;168:913-7.

29. Vinagre AL, Souza MV. Levothyroxine absorption and difficult management of hypothyroid patients in the intensive care unit: two case reports and a literature review [Article in English, Portuguese]. Rev Bras Ter Intensiva. 2011;23:242-8.

30. Centanni M, Gargano L, Canettieri G, et al. Thyroxine in goiter, Helicobacter pylori infection, and chronic gastritis. N Engl J Med. 2006;354:1787-95.

31. Lahner E, Virili C, Santaguida MG, et al. Helicobacter pylori infection and drugs malabsorption. World $\mathrm{J}$ Gastroenterol. 2014;20:10331-7.

32. Centers for Medicare and Medicaid Services. Physician fee schedule search. 2015. https://www.cms.gov/apps/physician-feeschedule/search/search-criteria.aspx. Accessed 2 April 2016.

33. Endocrinology 2014. Deductible plan members or non-covered services. http://info.kaiserpermanente.org/.../endocrinology_fee_ list.pdf. Accessed 4 April 2016.

34. Agency for Healthcare Research and Quality. HCUP-US NIS overview. http://www.hcupnet.ahrq.gov. Accessed 2 April 2016.

35. Blue Cross Blue Shield of Massachusetts. Typical costs for common medical services. http://www.bluecrossma.com/blue-iq/ pdfs/TypicalCosts_89717_042709.pdf. Accessed 5 April 2016.

36. Red Book online (database online). Greenwood Village, CO: Truven Health Analytics. http://www.micromedexsolutions.com. Accessed 3 Aug 2015.

37. Food and Drug Administration. Office of Public Health Strategy and Analysis. The public health evidence of FDA oversight of laboratory tests: 20 case studies. 2015. http://www.fda.gov/ downloads/AboutFDA/ReportsManualsForms/Reports/UCM472 777.pdf. Accessed 2 April 2016.

38. Ray KN, Chari AV, Engberg J, et al. Opportunity costs of ambulatory medical care in the United States. Am J Manag Care. 2015;21:567-74

39. Shaw MK, Davis SA, Fleischer AB, Feldman SR. The duration of office visits in the United States, 1993 to 2010. Am J Manag Care. 2014;20:820-6.

40. Mijailovic AS, Tanasijevic MJ, Goonan EM, et al. Optimizing outpatient phlebotomy staffing: tools to assess staffing needs and monitor effectiveness. Arch Pathol Lab Med. 2014;138:929-35.

41. IMS Institute for Healthcare Informatics. Medicine use and shifting costs of healthcare. Danbury: IMS Health; 2014. p. 46.

42. Irving SA, Vadiveloo T, Leese GP. Drugs that interact with levothyroxine: an observational study from the Thyroid Epidemiology, Audit and Research Study (TEARS). Clin Endocrinol (Oxf). 2015;82:136-41.

43. McMillan M, Rotenberg KS, Vora K, et al. Comorbidities, concomitant medications, and diet as factors affecting levothyroxine therapy: results of the CONTROL Surveillance Project. Drugs R D. $2016 ; 16: 53-68$. 
44. Hallert E, Husberg M, Kalkan A, et al. Early rheumatoid arthritis 6 years after diagnosis is still associated with high direct costs and increasing loss of productivity: the Swedish TIRA project. Scand J Rheumatol. 2014;43:177-83.

45. Owens GM. Managed care implications in managing rheumatoid arthritis. Am J Manag Care. 2014;20(7 Suppl.):S145-52.

46. Sun P, Peng X, Sun S, et al. Direct medical costs and medication compliance among fibromyalgia patients: duloxetine initiators vs. pregabalin initiators. Pain Pract. 2014;14:22-31.

47. Rachana PR, Anuradha HV, Shivamurthy MC. Anti hypertensive prescribing patterns and cost analysis for primary hypertension: a retrospective study. J Clin Diagn Res. 2014;8:HC19-22.

48. Ross DS, Daniels GH, Gouveia D. The use and limitations of a chemiluminescent thyrotropin assay as a single thyroid function test in an out-patient endocrine clinic. J Clin Endocrinol Metab. 1990;71:764-9.

49. Parle JV, Franklyn JA, Cross KW, et al. Thyroxine prescription in the community: serum thyroid stimulating hormone level assays as an indicator of undertreatment or overtreatment. $\mathrm{Br} \mathrm{J}$ Gen Pract. 1993;43:107-9.

50. Canaris GJ, Manowitz NR, Mayor G, Ridgway EC. The Colorado thyroid disease prevalence study. Arch Intern Med. 2000;160:526-34.

51. Vaisman F, Coeli CM, Ward LS, et al. How good is the levothyroxine replacement in primary hypothyroidism patients in Brazil? Data of a multicentre study. J Endocrinol Invest. 2013;36:485-8. 